\title{
Equalisation of blocking probability in switching systems with limited availability
}

\author{
M. Stasiak, M. Gtabowski, P. Zwierzykowski \\ Institute of Electronics and Telecommunications \\ Poznan University of Technology \\ ul. Piotrowo 3a, 60-965 Poznan, Poland
}

Phone: +486187822 92, Fax: +48618782572

E-mail: (stasiak, pzwierz,mglabows)@et.put.poznan.pl 1

\begin{abstract}
Three reservation algorithms for the limited-availability system are proposed and compared in the paper. These algorithms can be used for call blocking equalisation in outgoing links of multiservice switching networks. Two approximate methods of equalised blocking probability calculation in the system with limited availability are proposed. Results of analytical calculations are compared with results of digital simulation of limited availability groups and switching networks with reservation. These researches have confirmed a great accuracy of the proposed calculation methods. The formulae derived by the authors can be useful for the analysis and design of ISDN and B-ISDN systems.
\end{abstract}

Keywords

Bandwidth reservation, blocking probability, multi-rate model, switching network. 
Basic problems associated with the description of future B-ISDN (Broadband Integrated Services Digital Network) systems result from the necessity of servicing various types of traffic sources by the network. In principle, the classification of traffic sources in a broadband network is reduced to distinguishing the CBR (Constant Bit Rate) sources and the VBR (Variable Bit Rate) sources.

To define the loads introduced into networks by the VBR sources, it is proposed to determine the so-called equivalent bandwidth for particular classes of traffic streams generated by the sources (COST 224, 1992), (COST 242, 1996). The assignment of several constant bit rates to the VBR sources enables the evaluation of traffic characteristics of switching systems in the B-ISDN network by means of multi-rate models worked out for the multi-rate circuit switching. The multi-rate system services independent call demands with an integer number of basic bandwidth units. In circuit-switched network, the bandwidth unit is well defined as time-slot or channel. In (COST 242, 1996), (Bean, 1994), (Kawashima, 1986), (Korner, 1989), (Lindberger, 1987), (Roberts, 1983), (Takagi, 1988), (Theberge, 1995), (Tran-Gia, 1993) the multi-rate models are used to calculate the blocking probability in the full-availability group with reservation.

In the B-ISDN network the reservation mechanism is combined with the CAC function (Call Admission Control) (COST 224, 1992), (COST 242, 1996). The effectiveness of CAC function depends on the adopted access control strategy for different calls. One of the possible strategies is bandwidth reservation that assures maximum equalisation of blocking probability in a system for all streams of offered traffic (Roberts, 1983), (Tran-Gia, 1993).

The full-availability group is a discrete link model that uses complete sharing policy. In (Kaufman, 1981) and (Roberts, 1981) it has been proved that the multidimensional service process occurring in the full-availability group can be reduced to the one-dimensional Markov chain. Such reduction is the base for the determination of occupancy distribution in the group by means of a simple recurrent formula which is known as the Kaufman-Roberts recursion.

The calculation algorithm of more complicated systems consists in the approximation of a multi-dimensional service process by the one-dimensional Markov chain characterised by a product form solution (Beshai, 1988), (Roberts, 1983), (Stasiak, 1993a), (Stasiak, 1993b). Such approach leads to a simple formula for recurrence calculation of the occupancy distribution in multi-rate systems. This formula is a generalisation of the Kaufman-Roberts recursion.

In Section 2 the generalised Kaufman-Roberts recursion has been analysed. The full-availability group with reservation has been described in Section 3. In Section 4 a model of the limited-availability group with reservation has been considered. Limited-availability groups were the subject of many professional analyses, e.g. (Conradt, 1985), (Karlsson, 1991), (Lutton, 1984), (Ramaswami, 1985). In (Stasiak, 1993b), a simple approximate method of calculating the blocking 
probability in these groups is quoted. Solutions associated with reservation algorithms in the limited-availability group have not been proposed. The problem of introducing the reservation mechanism to this group is important. The outgoing links of switching networks can be treated as limited-availability groups (Stasiak, 1996). Thus, solutions concerning these groups are relevant for determining the conditions of blocking probability equalisation in switching networks.

The main aim of the present paper was to elaborate reservation algorithms for the limited-availability group. In Section 4 three reservation algorithms in the limited-availability group have been proposed. These algorithms have ensured blocking equalisation in the system for all traffic streams.

In Section 4 two approximate methods of blocking probability calculation in the limited-availability group with reservation have been derived. The results of analytical calculations have been compared with the results of digital simulation. In Section 5 problems of external blocking probability equalisation in switching networks have been discussed. Section 6 concludes the paper.

\section{GENERALISED KAUFMAN - ROBERTS MODEL OF THE MULTI - RATE SYSTEM}

Let us consider a multi-rate system with a capacity of $V$ bandwidth units. The system services $M$ independent classes of Poisson traffic streams with the intensities: $\lambda_{1}, \lambda_{2}, \ldots, \lambda_{M}$. The holding time for the calls of particular classes has an exponential distribution with the parameters: $\mu_{1}, \mu_{2}, \ldots, \mu_{M}$. A class $i$ call requires $t_{i}$ bandwidth units to set up a connection. Thus the mean traffic offered to the system by the class $i$ traffic stream is equal to:

$\alpha_{i}=\lambda_{i} / \mu_{i}$

\subsection{Basic recurrence relations}

The state of the system is determined by an ordered set: $\Omega=\left\{x_{1}, \ldots, x_{M}\right\}$. Each of those elements is equal to the number of given-class calls carried by the system. The probability of this state is designated by the symbol $p\left(x_{1}, \ldots, x_{M}\right)$. The total number of busy bandwidth units in the system is equal to:

$$
n=\sum_{i=1}^{M} x_{i} t_{i}
$$

The probability of $n$ basic bandwidth units being busy is denoted by the symbol $P(n)$. Figure 1 shows a fragment of the multi-dimensional process occurring in the 


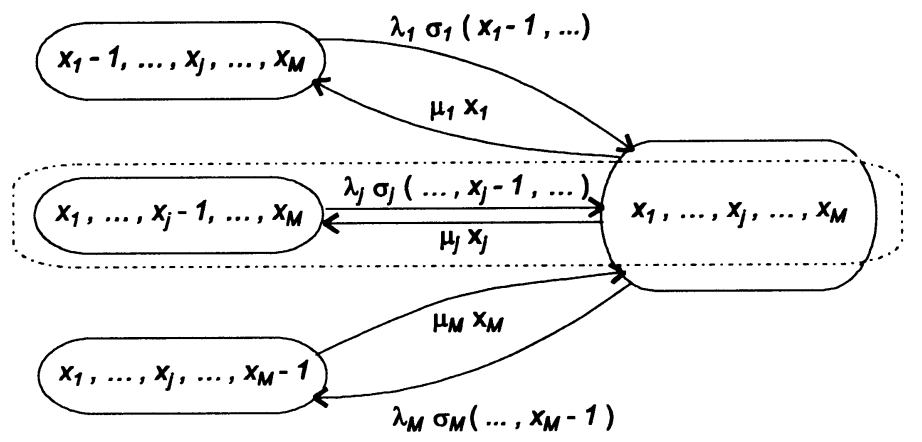

Figure 1 Multi-dimensional Markov process in the multi-rate system.

system under consideration. Let us consider the local equation of equilibrium associated with class $i$ stream (the states designated with a dash line in Figure 1):

$x_{i} \mu_{i} t_{i} p\left(x_{1}, \ldots, x_{i}, \ldots, x_{M}\right)=\lambda_{i} t_{i} \sigma_{i}\left(x_{1}, \ldots, x_{i}-1, \ldots, x_{M}\right) p\left(x_{1}, \ldots, x_{i}-1, \ldots, x_{M}\right)$

where $\sigma_{i}\left(x_{1}, \ldots, x_{i}-1, \ldots, x_{M}\right)$ is the conditional probability of passing between adjacent states of the process associated with the class $i$ stream. The value of the parameter $\sigma_{i}$ can be changed, depending on the state of the system. So, various solution are possible for equation (3), and consequently, the local birth and death processes associated with the relevant streams of calls will be mutually dependent. In (Beshai, 1988), (Stasiak, 1993a), (Stasiak 1993b) it was assumed that those dependencies are negligible when the following assumptions are fulfilled:

$\sigma_{i}\left(x_{1}, \ldots, x_{M}\right)=\sigma_{i}(n)$

$\left|\frac{\sigma_{i}(n)-\sigma_{i}(n-1)}{\sigma_{i}(n-1)}\right|<<1$

The assumption (4) means that $\sigma_{i}(n)$ does not depend on the division of the busy units between particular classes of calls. The assumption (5) means that $\sigma_{i}(n)$ is a slowly-varying function of $n$. Under such circumstances we can assume (Beshai, 1988) that the mutual dependence of service processes of particular classes is negligible. The assumptions (4) and (5) allow us to sum unconditionally all the $M$ type (3) equations for the state $\Omega=\left\{x_{1}, \ldots, x_{M}\right\}$ (Figure 1). Regarding the formula (1) as the result of such procedure we obtain:

$n p\left(x_{1}, \ldots, x_{i}, \ldots, x_{M}\right)=\sum_{i=1}^{M} a_{i} t_{i} \sigma_{i}\left(n-t_{i}\right) p\left(x_{1}, \ldots, x_{i}-1, \ldots, x_{M}\right)$ 


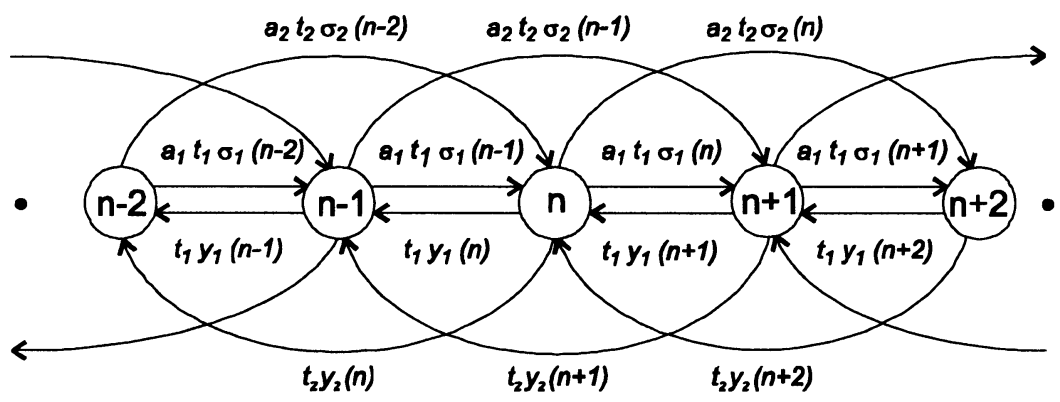

Figure 2 One-dimensional Markov chain.

Now, summing both sides of (6) for all sets $\Omega$ satisfying the equation (2) we finally obtain the so-called generalised Kaufman-Roberts recursion (Beshai, 1988), (Roberts, 1983), (Stasiak, 1993a):

$n P(n)=\sum_{i=1}^{M} a_{i} t_{i} \sigma_{i}\left(n-t_{i}\right) P\left(n-t_{i}\right)$

It has been mentioned that $P(n)$ is the state probability in the multi-rate system, and $\sigma_{i}(n)$ is the conditional (state-dependent) probability of passing between the adjacent states of the process associated with the class $i$ call stream. Thus, the blocking probability $b(i)$ for the class $i$ stream can be written as follows:

$b(i)=\sum_{n=0}^{V-t_{i}} P(n)\left[1-\sigma_{i}(n)\right]+\sum_{n=V-t_{i}+1}^{V} P(n)$

On the basis of the equations (7) - (8) we can approximately calculate the blocking probability in a state-dependent multi-rate system. However, for this purpose the probabilities $\sigma_{i}(n)$ have to be determined. It should be emphasised that the accuracy of calculating the distribution (7) depends on the level of accordance between the determined parameter $\sigma_{i}(n)$ and the assumptions (4) and (5).

It should be mentioned here that the presented analytical approximation (7) of the occupancy distribution in a multi-rate system results from the reduction of the multi-dimensional Markov process in the multi-rate system (Figure 1) to the one dimensional approximate Markov chain (Figure 2). The diagram presented in Figure 2 is appropriate to the generalised Kaufman-Roberts recursion (7) for the system with two call streams $\left(M=2, t_{1}=1, t_{2}=2\right)$. The $y_{i}(n)$ symbol denotes the reverse transition rates of a class $i$ service stream outgoing from state $n$.

If the probabilities of passing $\sigma_{i}(n)$ are equal to one for all states, equations (7) are reduced to the Kaufman-Roberts recursion (Kaufman, 1981), (Roberts, 1981): 


$$
n P(n)=\sum_{i=1}^{M} a_{i} t_{i} P\left(n-t_{i}\right)
$$

Equation (9) determines exactly the occupancy distribution in the full-availability group with different multi-rate traffic streams. The full-availability group is a discrete link model that uses complete sharing policy (COST 242, 1996). This system is an example of a state-independent system in which the passage between two adjacent states of the process associated with a given class stream does not depend on the number of busy bandwidth units in the system.

\subsection{Reverse transition rates}

A part of the one-dimensional Markov chain diagram constructed for two call streams according to equation (7) is shown in Figure 2. The $y_{i}(n)$ symbol denotes the reverse transition rates of a class $i$ service stream outgoing from state $n$. These transition rates are equal to the average number of class $i$ calls serviced in state $n$. Each state of the one-dimensional Markov chain in the multi-rate system (Figure 2) satisfies the following state equation:

$$
P(n)\left[\sum_{i=1}^{M} a_{i} t_{i} \sigma_{i}(n)+\sum_{i=1}^{M} t_{i} y_{i}(n)\right]=\sum_{i=1}^{M} a_{i} t_{i} \sigma_{i}\left(n-t_{i}\right) P\left(n-t_{i}\right)+\sum_{i=1}^{M} t_{i} y_{i}\left(n+t_{i}\right) P\left(n+t_{i}\right)
$$

From equation (7) it results that the sum of service streams outgoing from a state $n$ is equal to $n$ :

$$
n=\sum_{i=1}^{M} t_{i} y_{i}(n)
$$

According to formulae (7) and (11), equation (10) can be rewritten as follows:

$$
\sum_{i=1}^{M} a_{i} t_{i} \sigma_{i}(n) P(n)=\sum_{i=1}^{M} t_{i} y_{i}\left(n+t_{i}\right) P\left(n+t_{i}\right)
$$

Expression (12) is the equation of statistical equilibrium between the total stream outgoing from state $n$ towards higher states and the total service stream entering state $n$ from higher states. This equation holds only when the local balance equations for call streams of particular traffic classes are satisfied (Kaufman, 1981), (Stasiak, 1993a), (Stasiak, 1993b):

$$
a_{i} t_{i} \sigma_{i}(n) P(n)=t_{i} y_{i}\left(n+t_{i}\right) P\left(n+t_{i}\right)
$$


Based on equation (13), the reverse transition rates are equal to:

$y_{i}\left(n+t_{i}\right)=\left\{\begin{array}{lll}a_{i} \sigma_{i}(n) P(n) / P\left(n+t_{i}\right) & \text { for } & n+t_{i} \leq V \\ 0 & \text { for } & n+t_{i}>V\end{array}\right.$

Formula (14) determines the average number of class $i$ calls serviced in the state $n+t_{i}$ of the multi-rate system. For $\sigma_{i}(n)=1$ formula (14) designates the reverse transition rates in the full-availability group.

\subsection{The full-availability group with reservation}

Let us consider a full-availability group with bandwidth reservation. This system allows us to equalise the blocking probability for all classes of traffic streams. For this purpose, the reservation threshold $Q_{i}$ for each traffic class is designated. The parameter $Q_{i}$ determines the borderline state of a system, in which servicing class $i$ calls is still possible. All states higher than $Q_{i}$ belong to the so called reservation space $R_{i}$, in which class $i$ calls will be blocked:

$R_{i}=V-Q_{i}$

According to the equalisation rule (COST 242, 1996), (Roberts, 1983), (TranGia, 1993), the blocking probability in the full-availability group will be the same for all call stream classes if the reservation threshold for all traffic classes is identical and equal to the difference between the total capacity of a group and the value of resources required by the call of maximum demands $t_{\max }$ :

$Q=V-t_{\max }$

The method of blocking probability calculation in the full-availability group with reservation, proposed in (Roberts, 1983), determines state probabilities by the recursion (7), in which the conditional probabilities $\sigma_{i}(n)$ are equal:

$\sigma_{i}(n)=\left\{\begin{array}{lll}0 & \text { for } & n>Q \\ 1 & \text { for } & n \leq Q\end{array}\right.$

The equalised blocking probability for all call classes can be calculated as follows:

$b(i)=\sum_{n=Q+1}^{V} P(n)$ 


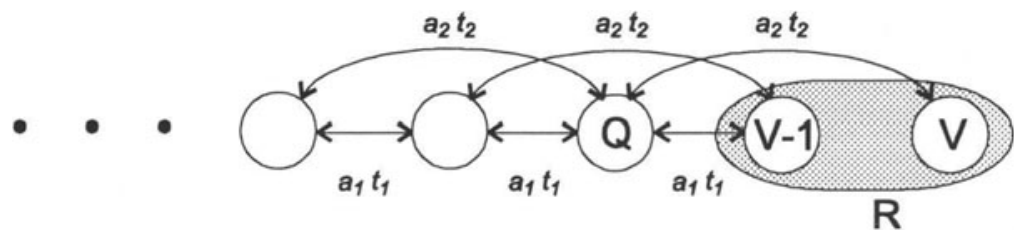

Figure 3 Markov chain conforming to full-availability group with reservation.

A diagram of the one-dimensional Markov chain according to the generalised Kaufman-Roberts recursion (7) with the probabilities of passing determined by equation (17) is shown in Figure 3. The diagram is appropriate to the fullavailability group with reservation. This group is offered two classes of traffic streams with bandwidth requirements: $t_{1}=1$, and $t_{2}=2$. The reverse transition rates are not marked in Figure 3 because these parameters are not necessary for the occupancy distribution calculation with the help of the equation (7). The calculation method of the reverse transitions has been presented in Section 2.2.

\section{THE LIMITED-AVAILABILITY GROUP WITH RESERVATION}

\subsection{Limited-availability group model}

The limited-availability group is a group divided into identical subgroups. The system services a call only when this call can be entirely carried by the resources of an arbitrary single subgroup. Let us consider a limited-availability group characterised by features of the following structural parameters (see Figure 4): $k$ - number of subgroups in a group, $f$ - capacity of a subgroup (the number of basic bandwidth units in a subgroup), $V$ - total capacity of a group $(V=k f)$.

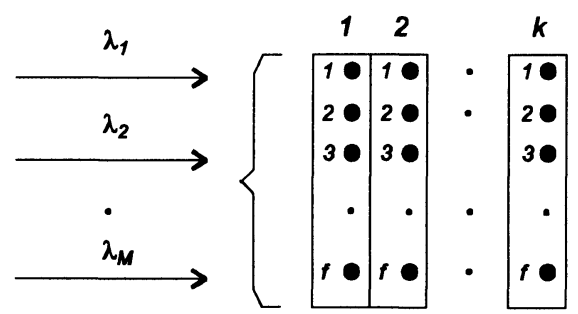

Figure 4 Limited-availability group

In (Stasiak, 1993b) a simple approximate method of calculating the blocking probability in these groups is proposed. According to this method, the state probabilities in the limited-availability group is approximated by the generalised 
Kaufman-Roberts recursion (7). The probability of passing $\sigma_{i}(n)$ in recursion (7) can be calculated as follows:

$$
\sigma_{i}(n)=\left[F(V-n, k, f)-F\left(V-n, k, t_{i}-1\right)\right] / F(V-n, k, f)
$$

where $F(x, k, f)$ is the number of arrangements of $x$ free bandwidth units in $k$ subgroups, calculated with the assumption that the capacity of each subgroup is limited to $f$ bandwidth units. The value of parameter $F(x, k, f)$ can be calculated by the following combinatorial formula:

$$
F(x, k, f)=\sum_{i=0}^{\left\lfloor\frac{x}{f+1}\right\rfloor}(-1)^{i}\left(\begin{array}{l}
k \\
i
\end{array}\right)\left(\begin{array}{c}
x+k-1-i(f+1) \\
k-1
\end{array}\right)
$$

The accuracy of the calculation of the occupancy distribution in the limitedavailability group (formulae (7) and (19)) depends on the degree of accordance of passing probabilities $\sigma_{i}(n)$ with the assumptions (4) and (5). The simulation tests (Stasiak, 1993b) carried out for various structures of limited-availability groups and for different mixtures of multi-rate traffic streams have confirmed great calculation accuracy of the formulae (7), (19) and (20). In practice, when $f \geq 5 t_{\max }$ (where $t_{\max }$ is the value of resources, required by a call of maximum demands), the results of calculations made in accordance with the mentioned formulae to determine the occupancy distribution in the limited-availability group can be regarded as accurate.

\subsection{Reservation algorithms in limited-availability group}

The blocking equalisation rule (16) does not take effect in the case of the limitedavailability group because of its structure and state-dependent processes occurring in the system. In this Section three reservation algorithms in the limitedavailability group are proposed. These algorithms make it possible to equalise well the blocking probabilities for all classes of offered traffic.

\section{Algorithm I}

In this algorithm we introduce the reservation threshold $Q$ for all call classes except for the oldest class $M$ (i.e. the one which requires the greatest number of bandwidth units to set up a connection: $t_{M}=t_{\max }$ ). This means that only class $M$ calls can be engaged by the system in the states belonging to the reservation space $R_{i}$. Thus the probability $\sigma_{i}(n)$ in recursion (7) can be expressed as follows: 


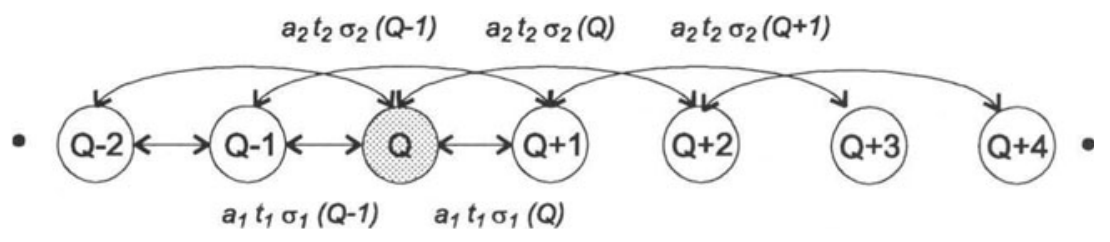

Figure 5 Markov chain in limited-availability group (reservation algorithm I).

$$
\begin{aligned}
& i \neq m \Rightarrow \\
& \sigma_{i}(n)=\left\{\begin{array}{cl}
{\left[F(V-n, k, f)-F\left(V-n, k, t_{i}-1\right)\right] / F(V-n, k, f)} & \text { for } n \leq Q \\
0 & \text { for } n>Q
\end{array}\right. \\
& i=m \Rightarrow \\
& \sigma_{i}(n)=\left[F(V-n, k, f)-F\left(V-n, k, t_{i}-1\right)\right] / F(V-n, k, f) \text { for each } n
\end{aligned}
$$

The $\sigma_{i}$ parameters in the states which do not belong to the reservation space are calculated in the same way as in the case of the limited-availability group without reservation. In the reservation space the conditional probabilities of passing are equal to zero, except for the oldest class stream. Thus, the blocking probability for class $M$ calls can be determined directly by (8). The blocking probability for class $i(i \neq M)$ calls can be calculated as follows:

$$
b(i)=\sum_{n=0}^{V-Q} P(n)\left[1-\sigma_{i}(n)\right]+\sum_{n=V-Q+1}^{V} P(n)
$$

A diagram of the Markov process according to the recurrence equation (7) for the limited-availability group with two offered call streams $\left(t_{1}=1, t_{2}=2\right)$ is shown in Figure 5. This diagram is appropriate for the reservation algorithm under consideration. The problem is to find the value of reservation threshold $Q$ in which the blocking probability of all traffic streams is equalised. The solution can be obtained by means of iterative method, according to the following plan. We accept the group capacity $V$ as the first value of reservation threshold $Q$. Then, on the base of the assumed $Q$ value, we calculate the occupancy distribution (equations (7), (21) and (22)) and the blocking probability for all traffic streams (equations (8) and (23)). Finally, we calculate the $|B(i)-B(j)|$ value for each pair of traffic streams $i, j$. If $|B(i)-B(j)| / B(i) \leq \xi$ (where $\xi$ is the value of the assumed relative error), $Q$ is the value of reservation threshold which causes blocking equalisation. Otherwise the value of the $Q$ parameter is decreased by one, and the 
calculation cycle is repeated. The number of steps is equal to the value of the reservation threshold.

In Figure 7, calculation results are compared with digital-simulation results for a limited-availability group characterised by parameters $V=120, k=4$ and $f=30$. In this group three classes of multi-rate traffic are offered: $\left(a_{1}=48 \mathrm{Erl}, t_{1}=1\right)$, $\left(a_{2}=24 \mathrm{Erl}, t_{2}=2\right)$ and $\left(a_{3}=8 \mathrm{Erl}, t_{3}=6\right)$. The computational time for one $Q$ value does not exceed $1 \mathrm{sec}$. (PC Pentium 133). The simulation results of one $Q$ value have been obtained for five series with 100000 arrivals of class 3 in each of them. An estimated simulation time has taken about $20 \mathrm{~min}$. The simulation was carried out for a random and sequential hunting strategy of subgroups.

The simulation results are obtained with $95 \%$ confidence interval. The values of that interval are not plotted on the diagram for better clarity. However, for each case the interval is at least one order smaller than the simulation result. All the results are expressed in relation to the value of reservation space $R$.

In view of our research, it can be stated that for $R=4$, the reservation algorithm in the limited-availability group brings about the blocking probability equalisation for the first $\left(t_{1}=1\right)$ and the second $\left(t_{2}=2\right)$ class of traffic. The blocking probability $b(3)$ of a call stream of highest demands (i.e. the one that requires the greatest number of bandwidth units to set up a connection) decreases when the value of $R$ increases. This phenomenon is caused by servicing only the third class calls in the reservation space. Thus the system has fewer remaining free bandwidth units for streams of lower demands (the first and the second class streams).

This is the reason why the blocking probability for streams of lower demands increases $(b(1), b(2))$. The blocking probabilities of all traffic classes are equalised for $R=11$. Above this value, the blocking probability for the third class stream exceeds the blocking probability for streams of lower demands.

\section{Algorithm II}

In this algorithms we introduce the reservation threshold $Q$ for all classes of call streams. This means that calls of all classes can not be engaged by the system in the states belonging to the reservation space $R$. Thus, the conditional passage probabilities in the recursion (7) can be expressed as follows:

$$
\sigma_{i}(n)=\left\{\begin{array}{cc}
{\left[F(V-n, k, f)-F\left(V-n, k, t_{i}-1\right)\right] / F(V-n, k, f)} & \text { for } n \leq Q \\
0 & \text { for } n>Q
\end{array}\right.
$$

The $\sigma_{i}$ parameters in the states which do not belong to the reservation space are calculated in the same way as in the case of the limited-availability group without reservation. In the reservation space the probabilities of passing are equal to zero. The blocking probability for all call classes can be calculated by the formula (23). 


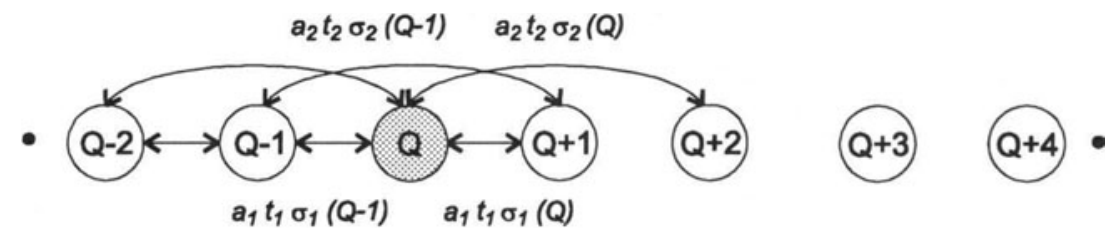

Figure 6 Markov chain in limited-availability group (reservation algorithm II).

A diagram of Markov process in a limited-availability group carrying two call streams is shown in Figure 6. This diagram is appropriate to the reservation algorithm under consideration. The reservation threshold $Q$ inducing the blocking probability equalisation can be found in the same iterative way as in the case of algorithm I described above.

In Figure 8 calculation results are compared with digital simulation results for a limited-availability group with the parameters determined above: $V=120, k=4$, $f=30, a_{1}=48 \mathrm{Erl}, t_{1}=1, a_{2}=24 \mathrm{Erl}, t_{2}=2, a_{3}=8 \mathrm{Erl}$ and $t_{3}=6$. With respect to our research, it can be stated that for $R \geq 4$, algorithm II leads to blocking probability equalisation for two lower call streams $\left(t_{1}=1, t_{2}=2\right)$. Blocking equalisation for all call classes takes places for $R \geq 15$, which is greater reservation space than in the case of algorithm I. The calls of the highest demands are not serviced in the reservation space. As a result of the above the blocking probability for the third class stream decreases slower than in the case of algorithm I. In the algorithm I, the calls of the third class are serviced in the reservation space. This means that the system which uses algorithm I is "broadened" for the third class calls. The inaccessibility of the reservation space in the case of algorithm II induces the system to become more and more "narrowed" for all class calls when the $R$ parameter increases. Due to this phenomenon, the value of equalised blocking probability increases slowly for $R>15$.

\section{Algorithm III}

It is possible to determine other reservation algorithms in the limited-availability group. Let us consider one of them. The algorithm proposed assumes designation of the reservation threshold for all traffic streams equal to $Q=f-t_{\max }$ in a certain number of subgroups. Thus, in the algorithm proposed we can distinguish the subgroups (from among $k$ subgroups) in which the reservation mechanism is introduced. The simulation results of the limited-availability group characterised by the parameters determined above $\left(V=120, k=4, f=30, a_{1}=48 \mathrm{Erl}, t_{1}=1\right.$, $a_{2}=24 \mathrm{Erl}, t_{2}=2, a_{3}=8 \mathrm{Erl}, t_{3}=6$ ) are shown in Figure 9. The results are expressed in relation to index $j$. This index determines the number of subgroups in which the reservation threshold is established. The reservation mechanism introduced into several subgroups causes the system to service more third class calls, and the blocking probability $b(3)$ decreases. Thus, the first and the second classes receive 


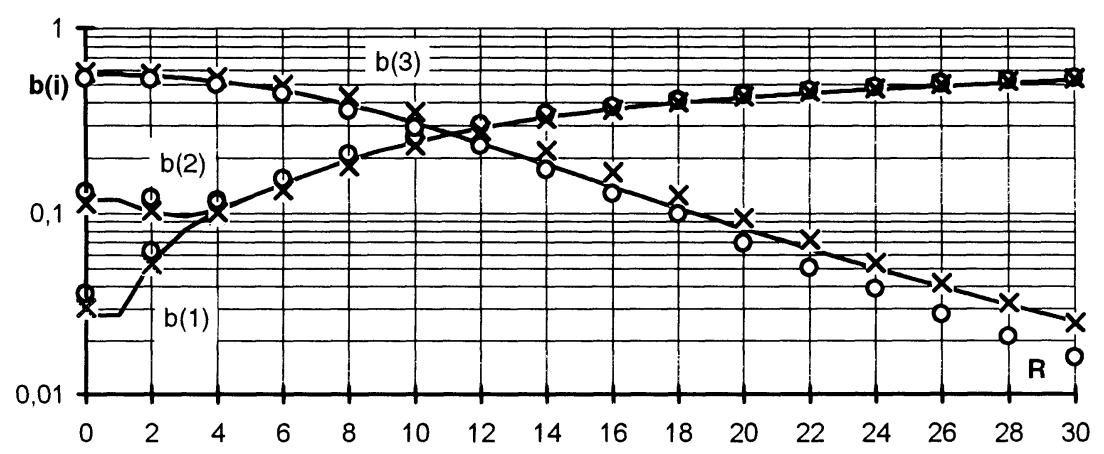

Figure 7 Blocking probability in the limited-availability group (algorithm I). Calculations: - Simulation: $x$ random strategy, o sequential strategy.

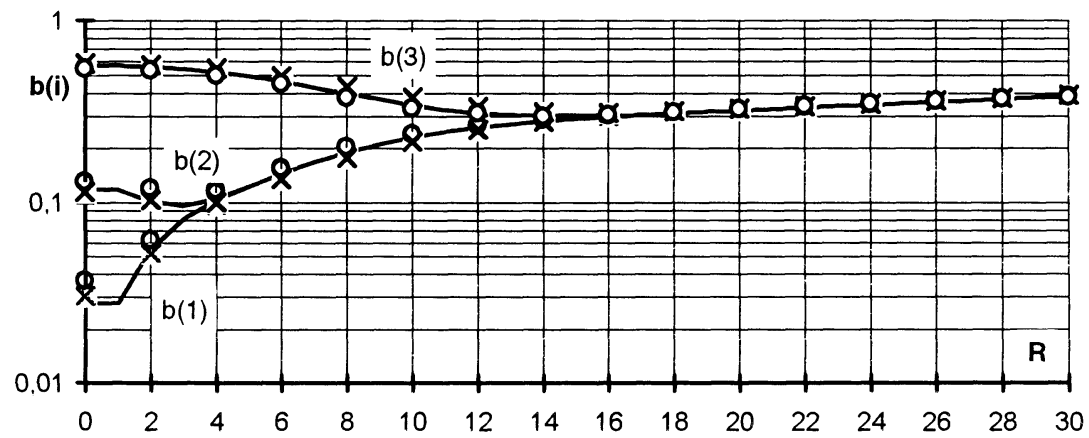

Figure 8 Blocking probability in the limited-availability group (algorithm II). Calculations: - Simulation: $\times$ random strategy, o sequential strategy.

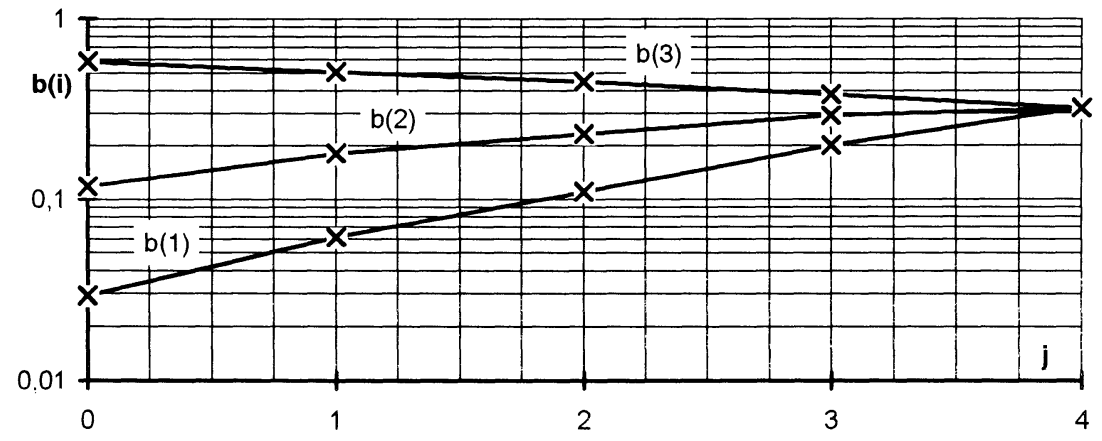

Figure 9 Blocking probability in the limited - availability group (algorithm III). Simulation: $-\times-$ random hunting strategy. 
fewer free bandwidth units, and the blocking probabilities $b(1)$ as well as $b(2)$ are increased. If reservation thresholds are introduced into all $k$ subgroups, the blocking probability of all traffic classes is equalised.

The elaboration of a simple method of the calculation of the blocking probability in the limited-availability group operating with reservation algorithm III is not possible. Contrary to the reservation algorithms I and II, the introduction of reservation threshold into several subgroups does not mean that the conditional probabilities of passing $\sigma_{i}(n)$ take the value of zero in the reservation space $R$. These parameters are different from zero and from the values determined by equation (19). This phenomenon results from the possibility of carrying call streams by the subgroups in which the reservation mechanism does not exist. Thus, the diagram of Markov process shown in Figure 2 is appropriate to the reservation algorithm under consideration.

The simulation research carried out by the authors indicate that in the case of introducing the reservation threshold into each subgroup the equalised blocking probability for the algorithm III can be approximated by the method developed for algorithm II (for the smallest value of reservation space $R$, in which blocking probabilities are equalised). For the majority of cases, such approximation does not include an error exceeding $5 \%$.

From an engineer's viewpoint the algorithm III can be considered as the most effective one. In ATM network, the CAC function tests a possibility of setting up a new connection at designated links or virtual paths. Thus, it seems to be technically sound to joint the reservation mechanism with the CAC function which checks the state of a given link or virtual path. Moreover, this solution allows the network operator to introduce the reservation mechanism into particular ATM links in accordance with the network configuration.

\section{SWITCHING NETWORKS WITH RESERVATION}

The third reservation algorithm can have a practical meaning for external blocking probability equalisation in multi-service switching networks. The outgoing link groups of switching networks can be treated as limited-availability group, in which particular links are regarded as subgroups (Stasiak, 1996). Figure 10 shows a three-stage switching network. The outgoing multiplexed transmission links create link groups called directions. The outgoing links can be wired to the directions in different ways. In Figure 10 each first link of each laststage switch belongs to the first direction. Analogously, each link $n$ of each laststage switch belongs to the same direction with serial number equal to $n$.

Blocking events in the switching network are the sum of internal and external blocking events. An internal blocking event is defined as the impossibility of setting up a connection between a given input and output link (or direction) of a switching network. In turn, external blocking event for class $i$ stream appears 


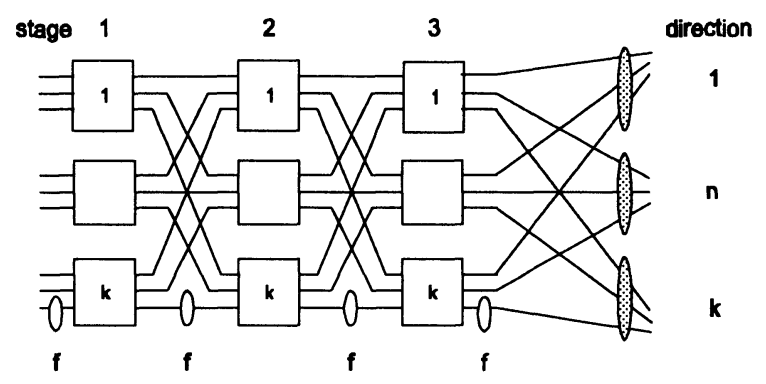

Figure 10 A three-stage switching network.

when no link of the given direction can carry a class $i$ call. In this paper only external blocking events are considered.

On the grounds of the limited-availability group definition (Section 4.1), it can be stated that the direction of the switching network can be regarded as the limited-availability group if only one link chosen arbitrarily from among other links belonging to this direction can carry a given class call. According to the third reservation algorithm, the reservation of $V-t_{\max }$ bandwidth units in each outgoing link of the direction leads to the equalisation of external blocking probabilities in this direction.

Figure 11 shows the simulation results of the total blocking probability in the switching network without reservation for all offered traffic streams. The simulations were carried out for a three-stage switching network consisting of the digital switches of $k \times k$ links. Each link has a capacity of $f$ bandwidth units. The outgoing links create $k$ identical directions, each with a capacity equal to $k$ links (Figure 10). Thus, the total capacity of direction expressed in bandwidth units is equal to $V=k f$. The simulations have been made for a switching network with the parameters $k=4$ and $f=30$. In this network three classes of multi-rate traffic in the following proportions $a_{1}: a_{2}: a_{3}=6: 3: 1$ are offered. The number of bandwidth units demanded for calls of particular classes are: $t_{1}=1, t_{2}=2, t_{3}=6$.

The simulation results are obtained with $95 \%$ confidence interval. The results are expressed in relation to the value of traffic offered to a single basic bandwidth unit, given by the formula:

$$
a=\sum_{i=1}^{M} a_{i} t_{i} / V
$$

Figure 12 shows the simulation results of the blocking probability in the switching network considered under the assumption that the third reservation algorithm is introduced into particular directions of the switching network. In consequence, blocking probabilities for all class streams approach one another. In comparison 


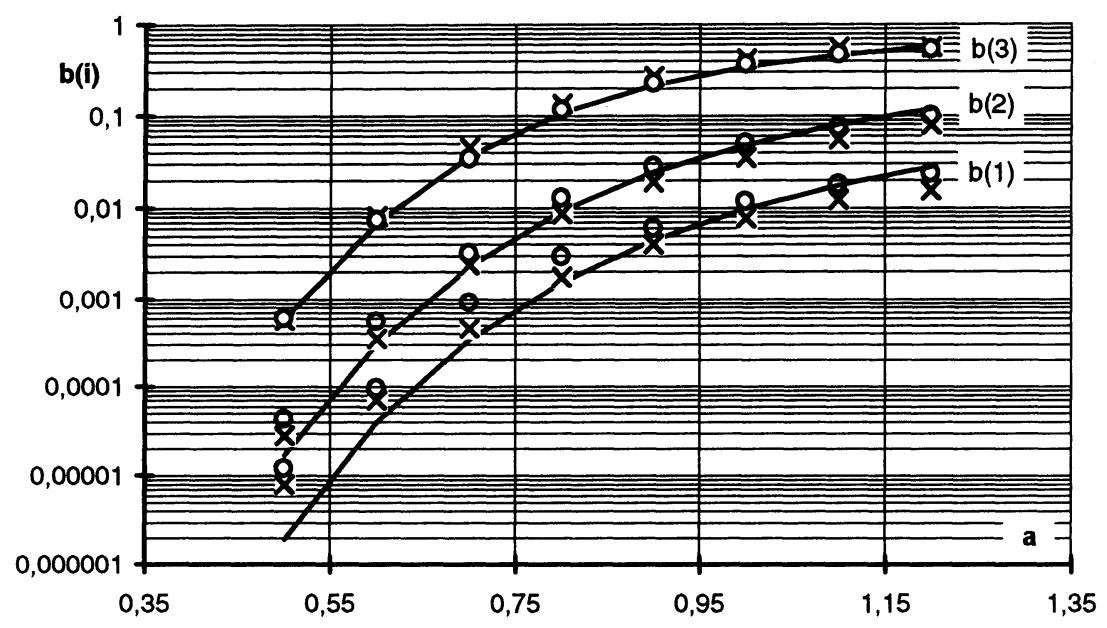

Figure 11 Blocking probability in the switching network without reservation. Simulation: $\times$ random hunting strategy, o sequential hunting strategy of links, Calculations (limited-availability group without reservation):

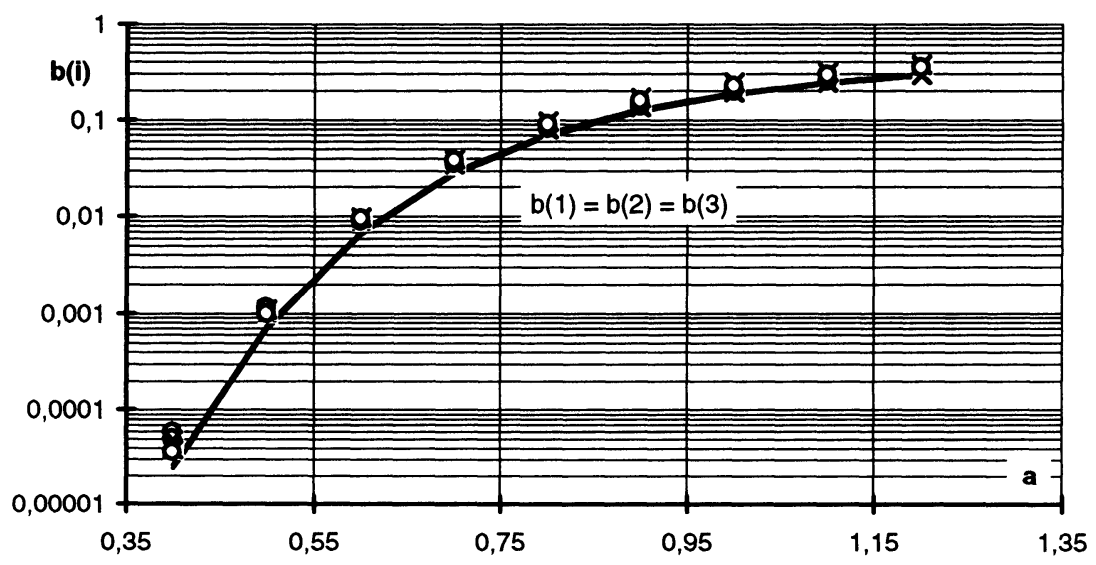

Figure 12 Blocking probability in the switching network with reservation. Simulation: $\times$ random hunting strategy, o sequential hunting strategy of links, Calculations (limited-availability group with reservation, algorithm II):

with Figure 11, the blocking probability $b(3)$ of the call stream of the highest demands decreases. This phenomenon is caused by carrying only the third class calls in the reservation space of each outgoing link. Thus the direction has fewer remaining free bandwidth units for streams of lower demands (the first and the second class streams) and the blocking probabilities $b(1)$ as well as $b(2)$ for 
streams of lower demands increases. The utilisation of the third algorithm in the outgoing directions of a switching network brings about the blocking probability equalisation for the first and the second class of traffic. Contrary to the limitedavailability group, the introduction of the third reservation algorithm into the outgoing directions of a switching network does not lead to the blocking probability equalisation for all traffic streams. Slight differences between the blocking probability $b(3)$ and the equalised probabilities $b(1), b(2)$ (Figure 12) result from the internal blocking of the switching network. In the structure of the switching network under consideration the internal blocking probability is negligible. Generally, however, equalisation of total blocking probability requires introduction of the reservation mechanism into inter-stage links of switching networks. The problem of internal blocking probability equalisation in switching networks is not considered in this paper.

To obtain a qualitative comparison of the limited-availability group with outgoing direction of switching network, Figure 11 and 12 show the results of blocking probability calculations in the limited-availability group with (and without) reservation. These calculation results were obtained on the basis of the methods discussed in Section 4.1 and Section 4.2.

In view of our research, it can be stated that the switching network characterised by a negligible number of internal blocking events can be calculated on the basis of limited-availability group models with (or without) reservation.

\section{CONCLUSION}

In the paper three reservation algorithms for the limited-availability group have been proposed. First two algorithms (I, II) assume independent strategies of subgroups occupation. They require one reservation mechanism for the system. The difference between algorithm I and II results from the determination of the reservation threshold for a traffic stream of the oldest class. In algorithm I this reservation threshold has not been introduced. In algorithm II, the reservation threshold for the oldest traffic class has been equal to the reservation threshold for other traffic classes. Algorithm III requires one reservation mechanism for each subgroup of the system. All algorithms proposed have led to the blocking probability equalisation in the limited-availability group.

In the paper, two methods of equalised blocking probability calculations have been derived; one method used for algorithm I and the other for algorithm II. The simulation tests have confirmed the validity of all the assumptions used in proposed methods. Equalised blocking probability in the limited-availability group resulting from algorithm III (reservation threshold is introduced into each subgroup) can be approximated by the method developed for algorithm II.

The proposed reservation algorithms can be useful for obtaining external blocking probabilities equalisation in the outgoing links of multi-service switching 
networks. From an engineer's viewpoint, algorithm III, assuming the introduction of the reservation mechanism into each subgroup, is the simplest one.

\section{REFERENCES}

***.: Performance evaluation and design of multiservice networks, Final Report COST 224, Published by the Commission of the European Communities (edited by Roberts J.W.), Brussels, 1992.

***.:Broadband Network Teletraffic, Final Report of Action COST 242, Published by the Commission of the European Communities (edited by Roberts J.W., Mocci V., Virtamo I.), Springer, Berlin, 1996.

Bean N.G., Gibbens R.J., Zachary S.: The performance of single resource loss systems in multiservice networks. Proc. 14th Int. Teletraffic Congress, Antibes Juan-les-Pins, 1994, vol. 1a, pp. 13-21.

Beshai M.E., Manfield D.R.: Multichannel services performance of switching networks, Proc. 12th Int. Teletraffic Congress, Torino, 1988, paper 5.1A.7.

Conradt J., Buchheister A.: Considerations on loss probability of multi-slot connections, Proc. 11th Int. Teletraffic Congress, Kyoto, 1985, paper 4.4B2.1.

Karlsson J.M.: Loss performance in trunk groups with different capacity demands, Proc. 13th Int. Teletraffic Congress, Copenhagen, 1991, vol. Discussion Circles, pp. 201-212.

Kaufman J.S.: Blocking in a shared resource environment, IEEE Trans. Commun., 1981, vol. COM 29, No. 10, pp. 1474-1481.

Kawashima K.: Trunk reservation models in telecommunication systems, Teletraffic Analysis and Computer Performance Evaluation, North Holland, Studies in Telecommunication, Amsterdam, 1986.

Korner U., Lubacz J., Pióro M.: Traffic engineering problems in multiservice circuit switched networks, Proc. 12th Int. Teletraffic Congress Specialist Seminar, Adelaide, 1989, paper 11.3.

Lindberger K.: Blocking for multislot heterogeneous traffic offered with reservation to a trunk group, Proc. 5th Int. Teletraffic Congress Seminar, Lake Como, 1987.

LuttonJ. L., Roberts J.W.: Traffic performance of multi-slot call routing strategies in an integrated services digital network, Proc. ISS"84, Florence, 1984.

Ramaswami V., Rao K.A.: Flexible time slot assignment - a performance study for the integrated services digital network, Proc. 11th Int. Teletraffic Congress, Kyoto, 1985.

Roberts J.W.: A service system with heterogeneous user requirements, in Performance of data communications systems and their applications, $G$. Pujolle (Ed.), North Holland Pub Co, Amsterdam, 1981. 
Roberts J.W.: Teletraffic models for the Telcom 1 Integrated Services Network, Proc. 10th Int. Teletraffic Congress, Montreal, 1983, paper 1.1. 2.

Stasiak M.: Blocking probability in a limited-availability group carrying mixture of different multichannel traffic streams, Ann. Telecommun., 1993, vol. 48, No. 1-2, pp. 71-76.

Stasiak M.: An approximate model of a switching network carrying mixture of different multichannel traffic streams, IEEE Trans. Commun., vol. COM-41, No. 6, 1993, pp. 836-840.

Stasiak M.: Combinatorial considerations for switching systems carrying multichannel traffic streams, Ann. Telecommun., 1996, No. 11-12, pp. 611-626

Takagi K., Sakita Y.: Analysis of loss probability equalized by trunk reservation for mixtures of several bandwidth traffic, Proc. 12th Int. Teletraffic Congress, Torino, 1988, paper 5.1. A. 1.

Theberge F., Mazumdar R.: On the Kaufman-Roberts recursion for large systems with reservations, Proc. First Workshop on ATM Traffic Management, Paris, 1995, pp. 51-58.

Tran-Gia P., Hubner F.: An analysis of trunk reservation and grade of service balancing mechanisms in multiservice broadband networks, Proc. Int. Teletraffic Congress Seminar: "Modeling and Performance evaluation of ATM technology", La Martynique, 1993.

\section{BIOGRAPHY}

Maciej Stasiak received the Ph.D. degree in electrical engineering from the Institute of Communications Engineering, Moscow, Russia, in 1984 and the Habilitation degree in electrical engineering from the Poznan University of Technology, Poland, in 1996. He is currently an associate professor at the Institute of Electronics and Telecommunications, Poznań University of Technology, Poland. His research interests are in the area of performance analysis and modelling multiservice networks and switching systems, in particular, resource allocation, call admission control, multi-rate systems and queuing systems.

Mariusz Glabowski received the M.Sc. degree in electrical engineering from the Poznań University of Technology, Poland, in 1997. He is currently a research assistant at the Institute of Electronics and Telecommunications, Poznań University of Technology, Poland. His research interests include ATM network, multi-rate models and resource allocation.

Piotr Zwierzykowski received the M.Sc. degree in electrical engineering from the Poznań University of Technology, Poland, in 1995. He is currently working towards Ph.D. degree at the Institute of Electronics and Telecommunications, Poznań University of Technology, Poland. His research interests include ATM network, multicast switching and switching networks. 